\title{
Identidad nacional y sus relaciones con la ideología y el bienestar en cinco países de fmérica Latina
}

\author{
Mational Identity and its Relations with Ideology and Well-Being in Five \\ Latin-fimerican Countries \\ Identidade nacional e suas relações com a ideologia e o bem-estar \\ em cinco países da fimérica Latina
}

\author{
Agustín Espinosa Pezzia*, Alessandro Soares Da Silva**, Carlos Contreras Ibáñez***, \\ Rosa María Cueto*, Aldo García Rengifo*, Fabio Ortolano**, \\ Juan Valencia****, Ángela Vera Ruíz* \\ *Pontificia Universidad Católica del Perú, **Universidade de São Paulo, \\ ***Universidad Autónoma Metropolitana-Iztapalapa, ****Universidad de Tarapacá
}

Doi: http://dx.doi.org/10.12804/revistas.urosario.edu.co/apl/a.3765

\section{Resumen}

Este estudio busca analizar las relaciones entre los componentes de la identidad nacional y la ideología política, en su manifestación autoritaria y de dominancia social, y las expresiones del bienestar subjetivo y social en muestras de cinco países de América Latina. Para tal fin, se desarrolló un estudio por encuestas en Brasil, Chile, Colombia, México y Perú ( $n=1039)$. Los resultados evidencian relaciones significativas entre las expresiones ideológicas con algunas dimensiones de la identidad. La ideología autoritaria se relaciona positivamente con los componentes identitarios, mientras que la ideología de dominancia social lo hace en menor medida y de manera inversa. Las relaciones entre los componentes de la identidad y el bienestar presentan una asociación positiva, y estas relaciones son más intensas en el caso del bienestar social que en el caso del bienestar subjetivo, aunque cierta heterogeneidad por país también es observada. Se intentó proponer un modelo general de las relaciones entre las variables derivadas de los tres constructos pero esta no alcanzó buenos niveles de ajuste, lo que se explicaría por la heterogeneidad de los resultados obtenidos por país. Palabras clave: identidad nacional, ideología política, bienestar, nacionalismo.

\section{fibstract}

This study seeks to analyze the relationships among the components of national identity, political ideology, in its authoritarian and social dominance manifestations

\footnotetext{
* Pontificia Universidad Católica del Perú (PUCP), Perú. Correo electrónico: agustin.espinosa@pucp.pe

** Universidade de São Paulo (USP), Brasil.

*** Universidad Autónoma Metropolitana-Iztapalapa (UAM-I), México.

**** Universidad de Tarapacá (UTA), Chile.

Cómo citar este artículo: Espinosa, A., Soares-da Silva A., Contreras, C., Cueto, R., García A., Ortolano, F., Valencia, J., \& Vera, A. (2017). Identidad nacional y sus relaciones con la ideología y el bienestar en cinco países de América Latina. Avances en Psicología Latinoamericana, 35(2), 351-374. doi: http://dx.doi.org/10.12804/revistas.urosario.edu.co/apl/a.3765
} 
and expressions of subjective and social well-being in samples from 5 countries of Latin America. To accomplish this aim, a survey research was conducted in Brazil, Chile, Colombia, Mexico and Peru $(\mathrm{n}=1039)$. Results show significant relationships between ideological expressions with some dimensions of identity. The authoritarian ideology is positively related to the identity components, whereas the social dominance ideology is inversely related to them. Relations among the components of identity and well-being have a positive association, and these relationships are more intense in the case of social well-being than in the case of subjective well-being. Nevertheless, some heterogeneity in these relations is observed by comparing countries. A general model of the relations among variables derived from three constructs was proposed, but this did not reach acceptable fit levels, being dismissed. It could be explained by the heterogeneity of the results by country. Keywords: National identity, Political Ideology, We1l-Being, Nationalism.

\section{Resumo}

Este estudo busca analisar as relações entre os componentes da identidade nacional e a ideologia política, em suas manifestações, autoritária e de dominância social; e as expressões do bem-estar subjetivo e social em amostras de cinco países da América Latina. Para tal fim, se desenvolveu um estudo por enquetes no Brasil, Chile, Colômbia, México e Peru ( $\mathrm{n}=1039)$. Os resultados evidenciam relações significativas entre as expressões ideológicas com algumas dimensões da identidade. A ideologia autoritária se relaciona positivamente com os componentes da identidade, enquanto que a ideologia de dominância social o faz em menor medida e de forma inversa. As relações entre os componentes da identidade e o bem-estar apresentam uma associação positiva, e estas relações são mais intensas no caso do bem-estar social que no caso do bem-estar subjetivo, ainda que certa heterogeneidade por país também seja observada. Tentou-se propor um modelo geral das relações entre as variáveis derivadas dos três conceitos, mas este não alcançou bons níveis de ajuste, o que se explicaria pela heterogeneidade dos resultados obtidos por país.

Palavras-chave: identidade nacional, ideologia política, bem-estar, nacionalismo.

\section{Nacionalismo e identidad nacional}

Desde las ciencias sociales, el concepto de nacionalismo propone distintas lecturas que van desde un análisis del conjunto de doctrinas ideológicas sobre una nación hasta un análisis de los sentimientos y el reconocimiento de pertenencia que realizan grupos de personas hacia esta (Ge1lner, 2008; Miles, 2000; Smith, 2004). Smith (2004) propone que ambas aproximaciones sobre el nacionalismo necesitan ser diferenciadas, pues a pesar de estar conceptualmente interrelacionadas, no siempre van de la mano. Sin embargo, otros autores intentan conciliar estas lecturas al referir que el nacionalismo alude a una identificación de carácter simbólico e ideológico con una nación (Anderson, 1993; Gellner, 2008; Herranz \& Basabe, 1999).

Independientemente de la postura académica que se asuma, hay consenso en establecer que los procesos de identificación con una nación y la construcción del nacionalismo están vinculados con un territorio de referencia, una cultura compartida y una memoria histórica sobre un origen común a un conjunto de personas (Anderson, 1993; Herranz \& Basabe, 1999; Miles, 2000; Salazar, 1996). En ese sentido, en el contexto latinoamericano, los elementos antes descritos confluyen ante la presencia de un Estado nación que actuará como categoría social (Béjar \& Capello, 1986; Gellner, 2008; Pérez, 1999; Salazar, 1996; Salazar \& Salazar, 1998).

La representación del nacionalismo como ideología otorga cierto nivel de agencia a los individuos que conforman una nación. Esto, si se asume que son ellos quienes por propia decisión establecen, promueven y/o aceptan tanto los aspectos materiales, representados por instituciones y roles, 
como los contenidos y símbolos adscritos a la concepción de determinada nación (Kaldor, 2004). Además, esta ideología permitiría a las personas desarrollar una identificación y un sentimiento de orgullo nacional (Pérez, 1999).

La concepción del nacionalismo como un proceso ideológico, subsume su funcionalidad en el ideal de alcanzar y mantener la autonomía, la cohesión y el fortalecimiento de la identidad de un conjunto de personas con las que se comparte la pertenencia a una categoría nacional (Smith, 2004). Sin embargo, para Gellner (2008), las ideologías nacionalistas sufren de una falsa conciencia, cuyos mitos distorsionan la realidad, pues ellas claman la defensa de la diversidad y las culturas populares, pero en la práctica producen una homogenización social bajo los criterios establecidos por ciertas élites sociales. Entonces, el mensaje central de la ideología nacionalista es que solo quienes aceptan dichos mensajes serán incluidos en una comunidad económica y moral (Gellner, 2008).

En relación con lo anterior, la tradición investigativa de las ciencias sociales ha relacionado esta representación ideológica con discursos de partidos políticos y élites intelectuales; y a pesar de la variabilidad de conceptos como 'sentimiento nacionalista' (Anderson, 1993), 'apego nacional' (Feshbach, 1987), 'patriotismo ciego' (Hurwitz \& Peffley, 1999) o 'nacionalismo excluyente' (Dekker, Malová \& Hoogendoorn, 2003), entre otros, las representaciones del nacionalismo y de la identificación con un endogrupo nacional parecen estar frecuente y estrechamente relacionadas con posiciones ideológicas conservadoras como el autoritarismo de ala derecha (Altemeyer, 2004).

El nacionalismo como fenómeno ideológico e identitario se podría expresar en manifestaciones prejuiciosas, xenofóbicas y beligerantes ante otros grupos nacionales (Dekker et al., 2003). En el contexto de América Latina, un ejemplo constantemente utilizado para entender la beligerancia entre grupos nacionales es el de la Guerra del Pacífico, que enfrentó a Perú y Bolivia con Chile hacia finales del siglo XIX. Este evento bélico podría considerarse la primera guerra que supone de manera explícita un enfrentamiento entre naciones y pone énfasis en el problema de las diferencias nacionales en América del Sur (Hosiasson, 2011). Por otro lado, algunas representaciones del nacionalismo son opuestas a los problemas descritos, encontrándose que las manifestaciones positivas del sentimiento nacional expresadas frecuentemente en el concepto de patriotismo se asocian con actitudes cívicas (Richey, 2011) y se expresan en interés, altruismo y sacrificio personal por el bienestar de la nación y sus miembros (Dekker et al., 2003).

Las representaciones expuestas tienen algunos puntos en común que traen de nuevo el escenario de la identificación nacional como condición necesaria por la cual se adquieren e internalizan los patrones de pensamiento y conducta referidos a la nación, así como un conjunto de criterios de comparación social con otros grupos relevantes (Blank, 2003). En síntesis, de las representaciones previas se desprenden un conjunto de sesgos que son consistentes con los supuestos de la Teoría de la Identidad Social (Tajfel, 1984), tales como la valoración positiva de la nación o sus miembros (Roccatto \& Sclauzero, 2002) y el despliegue de actitudes y conductas a favor de la propia nación y en contra de exogrupos relevantes (Dekker et al., 2003).

Entonces, en el estudio de los procesos de afiliación psicológica a grandes grupos como una nación, adquiere importancia el concepto de identidad social definido como "aquella parte del autoconcepto de un individuo que deriva del conocimiento de su pertenencia a un grupo social junto con el significado valorativo y emocional asociado con dicha pertenencia" (Tajfel, 1984, p. 292), pues la identidad nacional constituye un subtipo específico de identidad social (Nigbur \& Cinnirella, 2007; Pérez, 1999; Smith, Giannini, Helkama, Maczynski \& Stumps, 2005). Es decir que, por medio de sus instituciones, los sistemas de organización socio-política representados en 
Estados nación intentan dar respuesta a un conjunto de motivaciones o necesidades identitarias como la pertenencia, la autoestima, la diferenciación, el significado, la auto-eficacia y la continuidad (Simon, 2004; Vignoles, Regalia, Manzi, Golledge \& Scabini, 2006).

Algunas investigaciones han establecido que diversas expresiones de la identidad social influyen en el bienestar al facilitar la adaptación de los individuos a su medio (Haslam, Jetten, Postmes \& Haslam, 2009; Vignoles et al., 2006). La investigación sobre el bienestar en psicología ha sido abordada desde diversas aproximaciones (Rodríguez-Carvajal, Moreno-Jiménez, Blanco \& van Dierenonck, 2010). Una de las teorizaciones hegemónicas proviene de la tradición hedónica, donde se enfatizan aspectos afectivos como la felicidad y cognitivos como la satisfacción con la vida que las personas reportan. Un estudio transcultural intenta analizar cómo se relaciona el bienestar subjetivo de las personas con la valoración que estas hacen de la situación de su nación, encontrándose que la satisfacción con la nación fue una poderosa predictora de los niveles individuales de satisfacción con la vida, siendo esta relación moderada por el ingreso económico y las comodidades del hogar, el producto bruto per cápita y la región cultural del país (Occidente frente a no Occidente), entre otros (Morrison, Tay \& Diener, 2011). Los autores además encuentran que cuando los individuos están empobrecidos o se hallan limitados por su cultura, la satisfacción con la nación predecirá con mayor intensidad la satisfacción con la vida; en contraposición, las personas son más propensas a utilizar factores más próximos y tangibles para juzgar su satisfacción con la vida, cuando las condiciones sociales en las que se encuentran son positivas o cuando el individualismo como síndrome cultural es saliente (Morrison et al., 2011). De lo previamente revisado, es razonable afirmar que los procesos de identificación con un grupo nacional deberían relacionarse directamente con el bienestar de las personas.
En complemento, una segunda aproximación relevante para la comprensión del bienestar la provee el concepto de bienestar social (Keyes, 1998). De manera específica, el bienestar social alude a la relación que las personas establecen con su entorno y cómo este condiciona su bienestar a partir de cinco aspectos que son: (i) la integración social, (ii) la aceptación social, (iii) la contribución social, (iv) la actualización social y, (v) la coherencia social (Keyes, 1998). Las dimensiones descritas dan cuenta de que el bienestar estará estrechamente relacionado con la forma en que las personas establecen contacto social y relaciones interpersonales con otros individuos y grupos en sus sociedades (Blanco \& Díaz, 2005).

Desde la Teoría de la Identidad Social, se propone la idea de que las personas necesitan consolidar y mantener una autorepresentación positiva, como forma de asegurar la autoestima colectiva (Bar-Tal, 1996). De manera consistente con los procesos identitarios que involucran una categoría nacional, algunos estudios dan cuenta de que las personas que más se identifican con una categoría nacional, enfatizarán en la "esencialidad" de esta, relacionándola con autoestereotipos positivos (Keillor, Tomas \& Hult, 1999; Smith et al., 2005), pues las expectativas positivas sobre los miembros del endogrupo actúan como un fuerte elemento de cohesión (Van Vugt \& Hart, 2004).

\section{Nacionalismo e identidad nacional en los países de América Latina}

Los intentos por describir el sentimiento nacional y la identificación con endogrupos nacionales en América Latina suponen una tarea compleja debido a la diversidad social de los países de esta región. Así, retomando las reflexiones sobre el nacionalismo, la construcción de la identificación y el sentimiento nacional, se establece que estos elementos se constituyen sobre la referencia de símbolos y abstracciones socio-políticas y culturales hegemónicos, relacionados con los territorios 
que definen los límites geográficos de sus naciones (Anderson, 1993; Carteri, 2008; Espinosa, 2010; Vives, 1994) y en ocasiones estas representaciones al interior de un mismo país pueden resultar variadas e incluso excluyentes entre sí (Villas-Bôas, 2010; Vives, 1994).

Desde la psicología social, existen diversos abordajes psicosociales al estudio de la identidad nacional y el sentimiento nacional en los países de América Latina (Salazar \& Salazar, 1998). Una de estas aproximaciones se basa en la descripción y análisis de contenidos autoestereotípicos vinculados con las categorías nacionales en la región. Al respecto, los estudios sobre contenidos autoestereotípicos de los endogrupos nacionales realizados en las décadas del ochenta y noventa refieren al predominio de una autopresentación colectiva desvalorizada, lo que sugería la presencia de identidades nacionales negativas en grupos importantes de ciudadanos de países de América Latina (D'adamo \& García Beaudoux, 1995; Espinosa, 2003; Montero, 1996; Morales \& Páez; 1996). Estos resultados expresaban una representación deteriorada de los endogrupos nacionales y una cierta tendencia a valorar positivamente a exogrupos nacionales percibidos y valorados como de mayor competencia, desarrollo, instrumentalismo y estatus, en un fenómeno denominado altercentrismo (Montero, 1992). Posteriormente, estudios más recientes sobre identidad nacional en países de América Latina muestran que si bien, actualmente persisten los contenidos autoestereotípicos negativos, también han emergido contenidos autoestereotípicos positivos vinculados con estas naciones, lo que ha dado origen a representaciones identitarias donde la ambivalencia enfrenta a una antes generalizada negatividad (Beramendi \& $\mathrm{Zu}-$ bieta, 2013; Espinosa, 2010; Monsegur, Espinosa \& Beramendi, 2014; Roselli, 2000).

Esta reconfiguración de lo negativo a lo ambivalente en los autoestereotipos nacionales en América Latina respalda el hecho de que la construcción de la identidad y el sentimiento nacional siempre han sido un fenómeno contextualizado (Beramendi, 2014; Beramendi \& Zubieta, 2013; Espinosa, 2010). Es decir, las identificaciones sociales negativas de la década del noventa serían una consecuencia de los escenarios de crisis política, social y económica que muchos países de la región atravesaron durante esos años (Montero, 1996; Salazar \& Salazar, 1998). Mientras tanto, los estereotipos actuales suponen una revaloración de algunos contenidos negativos que se tornaron positivos, y el reconocimiento de otros tantos contenido positivos, aunque cierta negatividad persiste todavía (Espinosa, 2010; Monsegur et al., 2014).

Más recientemente, un estudio en Argentina, Brasil, Chile, Colombia, Perú y Venezuela muestra que las representaciones autoestereotípicas de los grupos nacionales en estos países podrían reducirse a cuatro dimensiones que han sido denominadas: calidez, competencia, moralidad y nacionalismo ideal (Espinosa, Acosta, Valencia et al., 2016). De manera específica, llama la atención la última dimensión referida que, de acuerdo con los autores, supone una visión cuestionadora y de desarrollo sobre los procesos de identificación nacional y construcción del sentimiento nacional, los cuales representan la imagen de conciudadanos comprometidos con sus países pero críticos con lo negativo que en estos sucede y, como se plantea en su discusión, esta dimensión pareciera estar contrapuesta a ideologías nacionalistas conservadoras de naturaleza autoritaria, que identificarían a los individuos críticos con su propia nación como amenazantes o socialmente desviados (Espinosa et al., 2016). Ahora bien, aunque esta dimensión comprende una asociación interesante de ideas sobre los miembros del endogrupo nacional, no es el atributo autoestereotípico con el que haya más acuerdo por parte de los participantes del estudio, independientemente de su país de origen (Espinosa et al., 2016).

No obstante lo anterior, las sutilezas de la relación entre los aspectos ideológicos e identitarios ha sido poco explorada desde la Psicología Social 
en América Latina. De manera específica, en el Perú estudios recientes con muestras de clase media dan cuenta de que ninguno de los componentes relacionados con la identificación con el país se asocian con el autoritarismo de ala derecha, aunque algunos valores asociados con el conservadurismo tradicional sí presentan relaciones pequeñas pero significativas con algunos aspectos positivos de la representación del endogrupo nacional (Espinosa, 2010). Lo anterior sugiere que el nacionalismo como ideología conservadora, autoritaria y excluyente no parece vincularse, al menos en muestras de clase media, con una adhesión mayor y cohesión endogrupal. Por otra parte, el espectro ideológico del conservadurismo expresado en la orientación a la dominancia social (Pratto, Sidanius, Stallworth \& Mallé, 1994) sí se relaciona con una representación negativa de los endogrupos nacionales en Perú (Espinosa, 2010) y Argentina (Monsegur et al., 2014), lo que parece estar vinculado con la autopercepción de los países de la región como grupos de bajo estatus (Espinosa, 2010; Monsegur et al., 2014). Lo anterior se puede explicar mediante el hecho de que personas que presenten una orientación hacia la dominancia social manifiesten valoraciones y afectos positivos hacia los grupos de alto estatus y un afecto negativo hacia los grupos de menor estatus, sin importar la adscripción identitaria del individuo (Levin \& Sidanius, 1999), siendo posiblemente unos de los antecedentes del fenómeno de altercentrismo previamente descrito.

Entonces, surge un debate interesante sobre los condicionantes ideológicos del nacionalismo y la identidad nacional, pues en los países de los que se ha podido recopilar información no se aprecia una expresión ni marcadamente excluyente o xenófoba relacionada con la ideología autoritaria de derecha, ni un aspecto marcadamente cívico o de interés en el bienestar de los miembros de los endogrupos nacionales expresados en la descripción positiva del patriotismo. Esto lleva a analizar la diversidad y la constitución de sociedades estamentales en los países de la región, donde es difícil desarrollar proyectos de identidad nacional inclusivos (Espinosa \& Cueto, 2014), y donde la orientación hacia la dominancia social (SDO) supone un elemento de desintegración social; con esto se evidencia que existe una relación inversa en la valoración de distintos grupos sociales y étnicos considerados de bajo estatus y la SDO en muestras de Perú (Espinosa, 2010) y Brasil (Pires, 2010).

A pesar de lo descrito, las relaciones entre ideologías conservadoras autoritarias o dominantes con la construcción del nacionalismo y la identidad nacional en América Latina no han sido descartadas o corroboradas de plano por la falta de investigación en muchos países de la región. Algo similar acontece con los estudios sobre las relaciones entre la identidad nacional y el bienestar en el contexto latinoamericano.

Sin embargo, retomando el estudio de Morrison et al. (2011), existen indicios para pensar que en países de América Latina con niveles de desarrollo social inferiores a los países que conforman el hemisferio occidental (EE. UU., Canadá y Europa Occidental) el nivel de satisfacción con el endogrupo nacional incrementará los niveles de bienestar subjetivo de las personas. Estudios en esta línea en Perú corroboran lo anterior (Espinosa \& Tapia, 2011; León, 2011), aunque, por ejemplo, esto no se corrobora en la Argentina (Espinosa, Beramendi \& Zubieta, 2012).

Desde otro abordaje del bienestar, existe evidencia en México (Laca-Arocena, Mejía \& Ibáñez, 2010) y Perú (Espinosa \& Tapia, 2011; León, 2011) de que las relaciones entre la identificación con el endogrupo nacional y los componentes del bienestar social son significativas de efecto medio $\mathrm{y}$ alto, aunque estas tampoco se encuentran en Argentina (Espinosa et al., 2012).

Con base en lo descrito, el presente estudio tiene como objetivos (I) describir y analizar las relaciones entre las ideologías conservadoras de tipo autoritario o dominante con los componentes de la identidad nacional en muestras de Brasil, 
Chile, Colombia, México y Perú, (II) describir y analizar la relación entre las expresiones del bienestar subjetivo y social con los componentes de la identidad nacional en los países descritos y (III) proponer un modelo general, que involucre a los cinco países listados, de las relaciones entre ideología, identidad nacional y bienestar.

A modo de hipótesis, se plantea una relación directa entre los niveles de autoritarismo de derecha y el grado de identificación, la autoestima colectiva y la autoestereotipia positiva ligada a cada endogrupo nacional. En contraste, la ideología de dominancia social se relacionará inversamente con los componentes de la identidad nacional descritos. Finalmente, manifestaciones positivas de los componentes de la identidad nacional se relacionarán positivamente con las dimensiones del bienestar evaluadas (ver tabla 1).

\section{Método}

\section{Participantes}

Participaron en el estudio 1039 personas de cinco países de América Latina (Brasil, Chile, Colombia, México y Perú). Del total de la muestra, el 59,7\% fueron mujeres. Las edades de los participantes fluctuaron entre los 14 y los 81 años $(M=23,44, D E=8,64)$. En todos los países las muestras fueron constituidas por ciudadanos de regiones urbanas. La mayoría de los participantes en el estudio se autocategorizaron como de nivel socioeconómico medio (52,6\%), un segundo grupo se autocategorizó como de nivel socioeconómico medio-alto (20,6\%), de cerca, en tercer lugar, estuvieron aquellos que se posicionaron como de nivel socioeconómico medio-bajo (20,4\%). En menor medida hubo participantes que se autocategorizaron como de nivel socioeconómico alto $(2,1 \%)$ y nivel socioeconómico bajo (2\%). Un $2,3 \%$ no se autoclasificó en ninguna categoría socioeconómica.

A pesar de la descripción anterior, es importante referir que algunas de las características demográficas de los participantes presentan ciertas diferencias entre países. Por tal motivo, en el siguiente cuadro se reportan de manera específica las características de cada muestra por país.

\section{Medidas e instrumentos de recolección de información}

Autoestima colectiva. Se utilizó la Subescala de autoestima colectiva privada de Luhtanen \&

Tabla 1

Caracteristicas demográficas de la muestra por país

\begin{tabular}{|c|c|c|c|c|c|c|c|}
\hline & & Brasil & Chile & Colombia & México & Perú & Total $100 \%$ \\
\hline \multicolumn{2}{|c|}{ Tamaño de la muestra } & $15715,1 \%$ & $24423,5 \%$ & $20319,5 \%$ & $20019,2 \%$ & $23522,6 \%$ & $1039100 \%$ \\
\hline \multirow{2}{*}{ Sexo } & hombre & $59 \%$ & $34,3 \%$ & $35,6 \%$ & $37,1 \%$ & $40,8 \%$ & $40,3 \%$ \\
\hline & mujer & $41 \%$ & $65,7 \%$ & $64,4 \%$ & $62,9 \%$ & $59,2 \%$ & $59,7 \%$ \\
\hline Edad & $\mathrm{M}(\mathrm{DE})$ & $24,25(9,82)$ & $20,87(2,99)$ & $33(12,47)$ & $22,09(3,13)$ & $18,63(2,59)$ & $23,44(8,64)$ \\
\hline \multirow{6}{*}{$\begin{array}{l}\text { Clase Social } \\
\text { Auto-percibida }\end{array}$} & alta & $0 \%$ & $0 \%$ & $1 \%$ & $1,5 \%$ & $7,2 \%$ & $2,1 \%$ \\
\hline & media-alta & $10,2 \%$ & $7,8 \%$ & $33,5 \%$ & $9 \%$ & $39,6 \%$ & $20.6 \%$ \\
\hline & media & $54,8 \%$ & $63,5 \%$ & $57,6 \%$ & $38 \%$ & $48,1 \%$ & $52,6 \%$ \\
\hline & media-baja & $27,4 \%$ & $25 \%$ & $7,4 \%$ & $41,5 \%$ & $4,3 \%$ & $20,4 \%$ \\
\hline & baja & $5,1 \%$ & $2 \%$ & $5 \%$ & $3,5 \%$ & $0 \%$ & $2,0 \%$ \\
\hline & no reporta & $2,5 \%$ & $1,6 \%$ & $0 \%$ & $6,5 \%$ & $0,9 \%$ & $2,3 \%$ \\
\hline
\end{tabular}


Crocker (1992). Esta subescala posee cuatro ítems que evalúan la relación afectiva de los participantes respecto de su identificación con su país. Las respuestas van del 1 al 5 en una escala tipo Likert, donde 1 es "totalmente en desacuerdo" y 5 es "totalmente de acuerdo". En el caso de Chile, Colombia, México y Perú, se utilizó la versión española de la escala con adaptaciones a las categorías nacionales correspondientes a cada país y validada en estudios previos en Argentina, Chile, Colombia, Perú y Venezuela (Beramendi, 2014; Espinosa, 2010; Espinosa et al., 2016; Monsegur et al., 2014). En el caso de Brasil, se utilizó la versión en portugués derivada de la validación de la versión en español antes descrita (Espinosa et al., 2016). La confiabilidad de la escala por países resultó muy buena en todos los casos: Brasil $(\alpha$ $=0,81)$, Chile $(\alpha=0,87)$, Colombia $(\alpha=0,86)$, México $(\alpha=0,80)$ y Perú $(\alpha=0,84)$.

Grado de identificación con el país (Espinosa, 2010). Se utilizó un ítem que mide el grado de identificación que posee un individuo con los miembros de su país. La pregunta se adaptó para cada categoría nacional. La escala de respuestas va del 1 al 5, donde 1 es "nada" y 5 es "total". En el caso brasileño se aplicó la versión del ítem en idioma portugués (Espinosa et al., 2016).

Autoestereotipos nacionales. La escala utilizada comprende una lista de 23 adjetivos positivos y sus respectivos antónimos, los cuáles buscan caracterizar qué tanto esos atributos son representativos de los miembros del endogrupo nacional. La versión utilizada de la escala es la desarrollada para la Argentina (Espinosa \& Beramendi, 2012) y validada y utilizada posteriormente en un estudio con seis países de América del Sur (Argentina, Brasil, Chile, Colombia, Perú y Venezuela) (Espinosa et al., 2016). Para el caso brasileño, la escala fue aplicada en su versión en idioma portugués (Espinosa et al., 2016). La escala de respuestas por ítem está estructurada como un diferencial semántico de siete puntos. Así, por ejemplo, los participantes debían marcar el número que más se acercara al adjetivo que creyeran era distintivo de las personas de su país. Entonces, en la dupla de los adjetivos solidarios-egoístas, 1 sería el acuerdo con que los miembros del endogrupo nacional son solidarios, 4 el acuerdo con que los miembros del endogrupo no son ni solidarios, ni egoístas y 7 sería el acuerdo con que los miembros del endogrupo son egoístas. Cabe resaltar que, como en procesos de investigación anteriores, los valores de la escala por ítem han sido invertidos para conseguir que la mayor puntuación en el atributo autoestereotípico corresponda a su valoración positiva; la menor puntuación correspondería a una valoración negativa del endogrupo nacional y puntuaciones intermedias significarían la falta de acuerdo sobre el aspecto positivo o negativo del atributo en relación con los miembros del endogrupo nacional (Espinosa et al., 2016). La escala comprende cuatro dimensiones autoestereotípicas: moralidad, competencia, calidez y nacionalismo ideal (Espinosa et al., 2016). Sobre esta versión se desarrollaron nuevas adaptaciones que comprenden la inclusión de un ítem (generosos frente a envidiosos) y la adaptación semántica de algunos adjetivos del cuestionario a las realidades de los cinco países participantes en el presente estudio. El ítem incluido ha sido incorporado a la dimensión moralidad. Los análisis de consistencia interna por país y por dimensión se reportan en la tabla 2.

Tabla 2

Alpha de Cronbach de dimensiones autoestereotípicas por país

\begin{tabular}{lccccc}
\hline & Brasil & Chile & Colombia & México & Perú \\
\hline Moralidad & 0,82 & 0,87 & 0,90 & 0,76 & 0,87 \\
Calidez & 0,85 & 0,80 & 0,82 & 0,80 & 0,73 \\
$\begin{array}{l}\text { Competen- } \\
\text { cia }\end{array}$ & 0,75 & 0,82 & 0,86 & 0,73 & 0,81 \\
$\begin{array}{l}\text { Nacionalis- } \\
\text { mo ideal }\end{array}$ & 0,53 & 0,40 & 0,64 & 0,10 & 0,43 \\
& & & & & \\
\end{tabular}


Orientación a la dominancia social. Para el caso de las muestras de países de habla hispana, se utilizó la versión validada por Cárdenas, Meza, Lagues y Yáñez (2010) en la población chilena. En el caso brasileño se utilizó una versión traducida al portugués de la versión antes descrita. La escala de respuesta del cuestionario para los casos de Chile, México y Perú fue del 1 al 6, donde 1 era "totalmente en desacuerdo" y 6 "totalmente de acuerdo" con las afirmaciones esbozadas en cada ítem. En los casos de Brasil y Colombia la escala de respuesta fue del 1 al 7, donde 1 significaba "total desacuerdo" y 7 "total acuerdo" con las afirmaciones presentadas en cada ítem. Para salvar las diferencias comprendidas en las escalas, se realizó una transformación por medio de una regla de tres sobre un valor máximo de 42 puntos en la escala general por cada país, lo que garantizó la posibilidad de comparar los resultados entre muestras. Los análisis de consistencia interna para la puntuación general de la escala por país fueron buenos: Brasil $(\alpha=0,83)$, Chile $(\alpha=0,82)$, Colombia $(\alpha=0,83)$, México $(\alpha=0,86)$ y Perú $(\alpha=0,88)$.

Autoritarismo de ala derecha. Se utilizó la versión abreviada de 12 ítems validada por Cárdenas y Parra (2010) en Chile. Una traducción de esta escala al portugués se realizó para ser aplicada en la muestra brasileña. La escala de respuesta a los ítems del cuestionario iba de 1 a 6 puntos, donde 1 reflejaba un "desacuerdo total" y 6 un "acuerdo total" con los enunciados propuestos en cada ítem. Los niveles de consistencia interna de la puntuación general de la escala por país fueron disímiles y en algún caso bajo: Brasil $(\alpha=0,62)$, Chile $(\alpha=$ $0,60)$, Colombia $(\alpha=0,76)$, México $(\alpha=0,43)$ y Perú $(\alpha=0,70)$.

Satisfacción con la vida. Se utilizó la versión en español adaptada y aplicada en el Perú (Martínez, 2006). Esta escala evalúa la dimensión cognitiva del bienestar subjetivo y está compuesta por cinco ítems con afirmaciones sobre la propia vida y con opciones de respuesta en una escala de 1 a 7 puntos, donde 1 significa un "absoluto desacuerdo" con la afirmación y 7 un "total acuerdo". El análisis de consistencia por país muestra confiabilidades de aceptables a buenas: Brasil $(\alpha=0,78)$, Chile $(\alpha=0,75)$, Colombia $(\alpha=$ $0,82)$, México $(\alpha=0,82)$ y Perú $(\alpha=0,73)$.

Bienestar social. Se utilizó la versión validada por Blanco y Díaz (2005) en España. Una traducción al portugués de dicha versión fue aplicada con la muestra brasileña. La escala está compuesta por 33 ítems, por medio de estos, los participantes evalúan distintos aspectos de su relación con el entorno social utilizando un formato de respuesta. En los casos de Brasil y Colombia consistió en una escala tipo Likert de 1 a 5 puntos, donde 1 supone un "total desacuerdo" con la afirmación esbozada en el ítem y 5 un "total acuerdo". En el caso de Chile, México y Perú la escala de respuesta fue de 1 a 7 , siguiendo el mismo patrón arriba descrito. Para salvar las diferencias comprendidas en las escalas, se realizó una transformación por medio de una regla de tres sobre un valor máximo de 35 puntos en la escala general por cada país, lo que garantizó la posibilidad de comparar los resultados entre muestras. Los análisis de consistencia interna por país y por dimensión se reportan en la tabla 3

Tabla 3

Alpha de Cronbach de dimensiones de bienestar social por país

\begin{tabular}{lccccc}
\hline & Brasil & Chile & Colombia & México & Perú \\
\hline $\begin{array}{l}\text { Integración } \\
\text { social }\end{array}$ & 0,80 & 0,74 & 0,75 & 0,68 & 0,73 \\
$\begin{array}{l}\text { Aceptación } \\
\text { social }\end{array}$ & 0,86 & 0,79 & 0,83 & 0,84 & 0,81 \\
$\begin{array}{l}\text { Contribución } \\
\text { social }\end{array}$ & 0,52 & 0,40 & 0,42 & 0,35 & 0,45 \\
$\begin{array}{l}\text { Actualización } \\
\text { social }\end{array}$ & 0,71 & 0,63 & 0,77 & 0,36 & 0,72 \\
$\begin{array}{l}\text { Coherencia } \\
\text { social }\end{array}$ & 0,32 & 0,57 & 0,60 & 0,28 & 0,69 \\
$\begin{array}{l}\text { Bienestar } \\
\text { social total }\end{array}$ & 0,84 & 0,84 & 0,85 & 0,78 & 0,87 \\
\hline
\end{tabular}




\section{Procedimiento}

El levantamiento de información en los cinco países se realizó entre los meses de mayo y julio de 2014. La recolección de datos se desarrolló de dos maneras, una presencial en contextos educativos, donde se aplicaba el cuestionario a estudiantes universitarios, y una virtual, por medio de la plataforma Google Docs, que permitía tener acceso a población general. En los casos de Chile, México y Perú, el levantamiento de información fue de manera presencial en población universitaria. En el caso de Brasil, el levantamiento fue presencial con población estudiantil universitaria y de formación profesional técnica. En el caso de Colombia, el levantamiento de información fue virtual con población general. Cabe resaltar que la utilización de una versión virtual puede resultar equivalente a una aplicación presencial (Lyons, Cude, Lawrence \& Gutter, 2005). La aplicación de los cuestionarios, en ambas versiones vino acompañada de un consentimiento informado que daba cuenta del objetivo general del estudio, el carácter voluntario de la participación en él, el respeto por la confidencialidad de las respuestas, la salvaguarda del anonimato de los participantes y el uso de la información con fines estrictamente académicos.

La información obtenida fue sometida a un procesamiento estadístico a partir de técnicas descriptivas, correlacionales, inferenciales y multivariadas pertinentes para los objetivos del estudio. Los análisis realizados consideran los niveles de consistencia interna de algunas de las medidas utilizadas; y aunque en general la confiabilidad de las escalas ha sido aceptable, en algunos casos se utilizarán coeficientes de consistencia interna regulares basados en lo propuesto por el metaanálisis de Mezulis, Abramson, Hyde y Hankin (2004), donde se encuentra que, en las escalas que incorporan algún criterio de autopresentación, existe cierta estabilidad y sesgo positivo en las respuestas, lo que suele reducir las confiabilidades, por lo que resulta apropiado trabajar hasta con coeficientes de consistencia interna de 0,50. Algunas excepciones en el análisis se harán con aquellos casos que se ubiquen por debajo de este valor, pero siempre considerando este aspecto en la discusión.

\section{Resultados}

\section{Análisis descriptivos, inferenciales y correlacionales de las medidas de ideología política}

En el total de la muestra, en una escala del 1 al 7 , el puntaje promedio en la medida de autoritarismo de ala derecha fue de 2,99 $(D E=0,66)$. Asimismo, los puntajes promedio por país en esta medida se reportan en la tabla 4.

Tabla 4

Media y desviación estándar de la medida de autoritarismo de ala derecha por país

\begin{tabular}{cccccc}
\hline & Brasil & Chile & Colombia & México & Perú \\
\hline $\mathbf{m}$ & 2,69 & 2,84 & 3,06 & 3,04 & 3,24 \\
\hline de & 0,64 & 0,59 & 0,79 & 0,53 & 0,61 \\
\hline
\end{tabular}

Un análisis de varianza muestra que existen diferencias por país en la puntuación promedio de autoritarismo de ala derecha, $F(4,997)=20,34, p$ $<0,001$. Posteriores análisis de Bonferroni señalan que la muestra peruana puntúa significativamente más alto que el resto de países en esta dimensión $\left(p_{s}<0,05\right.$ en las comparaciones con las muestras de Colombia y México y $p_{s}<0,001$ en las comparaciones con las muestras de Brasil y Chile). Un segundo grupo lo conforman Colombia y México, países que no se diferencian entre sí, pero que puntúan significativamente más alto que Brasil $\left(p_{s}<0,001\right.$ en ambos casos) y Chile $\left(p_{s}<0,01\right.$ en ambos casos). Un último grupo está conformado por las muestras de Brasil y Chile, que presentan las puntuaciones más bajas en el indicador de autoritarismo y no se diferencian entre sí. 
Luego, para el total de la muestra, en una escala del 6 a 42 puntos, el puntaje promedio en la medida de orientación a la dominancia social fue de 14,49 puntos $(D E=5.47)$. Los puntajes promedio de las muestras por país en dominancia social se reportan en la tabla 5 .

Tabla 5

Media y desviación estándar de la medida de orientación a la dominancia social por país

\begin{tabular}{lccccc}
\hline & Brasil & Chile & Colombia & México & Perú \\
\hline $\mathbf{m}$ & 16,09 & 11,94 & 16.65 & 12,94 & 15,55 \\
\hline de & 6,23 & 4 & 5,82 & 4,74 & 5,12 \\
\hline
\end{tabular}

Un análisis de varianza evidencia que existen diferencias en las muestras por país en la puntuación promedio de SDO, $F(4,982)=33,323, p<0,001$. Posteriores análisis de Bonferroni muestran que las muestras colombiana, brasileña y peruana no se diferencian entre sí y puntúan significativamente más alto que las muestras de Chile y México en esta medida ( $p_{s}<0,001$ en todos los casos). No se aprecian diferencias estadísticamente significativas entre las muestras de Chile y México.

Luego, se realizó un análisis de correlación de tipo Pearson entre las medidas de autoritarismo de ala derecha y dominancia social para el total de la muestra, encontrándose una relación de, $r(940)=$ $0,29, p<0,001$.

Las magnitudes de las correlaciones entre ambas medidas por país se reportan en la tabla 6 .

Tabla 6

Correlaciones tipo Pearson entre autoritarismo de ala derecha y orientación a la dominancia social por país

\begin{tabular}{lcclll}
\hline & Brasil & Chile & Colombia & México & Perú \\
\hline $\mathbf{r}$ & $0,46^{* * * *}$ & $0,37 * * *$ & $0,28 * * *$ & $0,18^{*}$ & $0,19 * *$ \\
\hline
\end{tabular}

$* * * \mathrm{p}<.001, * * \mathrm{p}<.01, * \mathrm{p}<.05$

Los resultados muestran relaciones significativas entre ambas medidas ideológicas en las muestras de todos los países, aunque estas relaciones difieren en las magnitudes por país.

\section{Análisis descriptivos e inferenciales de las medidas de identidad nacional}

En una escala del 1 al 5, el grado de identificación con el endogrupo nacional en la muestra de los cinco países es de $M=3,79 ; D E=1,10$, asimismo, en una escala del 1 al 5 , el nivel de autoestima colectiva en la muestra total es de $M=4,01 ; D E$ $=1$. A continuación se presentan los niveles de identificación y autoestima colectiva por país.

Tabla 7

Media y desviación estándar de las medidas de grado de identificación y autoestima colectiva por país

\begin{tabular}{lcccccc}
\hline & & Brasil & Chile & Colombia & México & Perú \\
\hline $\begin{array}{l}\text { Grado de } \\
\text { identifica- } \\
\text { ción }\end{array}$ & m & 3,50 & 3,74 & 4,14 & 3,45 & 3,92 \\
\hline $\begin{array}{l}\text { Autoesti- } \\
\text { ma colec- } \\
\text { tiva }\end{array}$ & m & 1,12 & 1,13 & 1 & 1 & 0,97 \\
\hline
\end{tabular}

En cuanto a los autoestereotipos colectivos, en una escala del 1 al 7, donde 1 es la representación negativa de la dimensión autoestereotípica y 7 la representación positiva de esta, la muestra general refleja los siguientes valores promedio para la dimensión de calidez, $M=5,31 ; D E=1,06$; competencia, $M=4,96 ; D E=1,06$; nacionalismo ideal, $M=4,09 ; D E=1,14$ y moralidad, $M=3,98$; $D E=1,05$. De manera específica, se presentan los promedios y desviaciones estándar de las dimensiones autoestereotípicas por país en la tabla 8 .

$\mathrm{El}$ análisis $\mathrm{T}$ de medidas repetidas comparando las puntuaciones de cada dimensión en la muestra general reflejan que la dimensión de calidez es la más consensual y se diferencia significativamente de la competencia, $t(1004)=12,89, p<0,001$; la moralidad, $t(996)=36,10, p<0,001$ y el nacionalismo ideal, $t(1017)=28,22, p<0,001$. La competencia aparece como el segundo atributo más valorado positivamente y se diferencia significativamente de la moralidad, $t(991)=31,41, p<0,001$ 
Tabla 8

Media y desviación estándar de las dimensiones del autoestereotipo nacional por país

\begin{tabular}{|c|c|c|c|c|c|c|}
\hline & & Brasil & Chile & Colombia & México & Perú \\
\hline \multirow{2}{*}{ Calidez } & $\mathrm{m}$ & 5,67 & 4,87 & 5,94 & 4,85 & 5,36 \\
\hline & de & 1,06 & 0,98 & 0,85 & 1,15 & 0,86 \\
\hline \multirow{2}{*}{$\begin{array}{l}\text { Competen- } \\
\text { cia }\end{array}$} & $\mathrm{m}$ & 4,72 & 4.62 & 5,70 & 4,45 & 5,25 \\
\hline & de & 0,93 & 0,97 & 0,99 & 1 & 0,90 \\
\hline \multirow{2}{*}{ Moralidad } & $\mathrm{m}$ & 3,74 & 4.10 & 4,44 & 3,76 & 3,80 \\
\hline & de & 0,94 & 0,99 & 1,16 & 0,96 & 1,01 \\
\hline \multirow{2}{*}{$\begin{array}{l}\text { Nacionalis- } \\
\text { mo ideal }\end{array}$} & $\mathrm{m}$ & 3,31 & 4,39 & 4,25 & 4,02 & 4,20 \\
\hline & de & 1,09 & 1,03 & 1,31 & 0,98 & 1,01 \\
\hline
\end{tabular}

y nacionalismo ideal, $t(1012)=23,10, p<0,001$. Finalmente, el nacionalismo ideal aparece como el tercer atributo con mayor acuerdo en la muestra general, diferenciándose significativamente de la moralidad, $t(1005)=3,24, p<0,001$. Los análisis por país replican parcialmente los resultados de la muestra general. Así, se corroboran las diferencias entre calidez y el resto de dimensiones en todos los países. Lo mismo sucede con la dimensión de competencia, cuya puntuación es la segunda más consensual en todas las muestras por país. Luego, en Brasil y Colombia la moralidad aparece como la tercera dimensión con mayor puntuación, mientras que en Chile, México y Perú esta tercera ubicación corresponde al nacionalismo ideal.

\section{Análisis descriptivos e inferenciales de las medidas de bienestar}

En una escala del 1 al 7, el nivel de satisfacción con la vida en la muestra de los cinco países es de $M=4,91 ; D E=1,10$, asimismo, en una escala de 5 a 35 puntos, la puntuación media de bienestar social en la muestra total es de $M=23,14 ; D E=2,86$. De manera específica, las puntuaciones medias en las dimensiones de la escala de bienestar social para el total de la muestra son las siguientes: integración social, $M=23,23 ; D E=4,46$, aceptación social,
$M=20,59 ; D E=4,67$, contribución social, $M=$ 23,53; $D E=3,88$, actualización social, $M=23,03$; $D E=4,48$ y coherencia social, $M=26 ; D E=3,98$.

La tabla 9 reporta las medias y desviaciones estándar por país en las medidas de bienestar.

Tabla 9

Media y desviación estándar de las medidas de bienestar

\begin{tabular}{|c|c|c|c|c|c|c|}
\hline & & Brasil & Chile & Colombia & México & Perú \\
\hline \multirow{2}{*}{$\begin{array}{l}\text { Satisfac- } \\
\text { ción con la } \\
\text { vida }\end{array}$} & $\mathrm{m}$ & 4,44 & 4,96 & 4,98 & 4,89 & 5,1 \\
\hline & de & 1,20 & 0,95 & 1,09 & 1,13 & 0,90 \\
\hline \multirow{2}{*}{$\begin{array}{l}\text { Bienestar } \\
\text { social total }\end{array}$} & $\mathrm{m}$ & 24,07 & 22,97 & 24,41 & 21,13 & 23,39 \\
\hline & de & $3 ; 22$ & 2,08 & 3,10 & 2,82 & 2,18 \\
\hline \multirow{2}{*}{$\begin{array}{l}\text { Integración } \\
\text { social }\end{array}$} & $\mathrm{m}$ & 24,36 & 21,27 & 26,31 & 22,95 & 22,19 \\
\hline & de & 5,07 & 3,29 & 4,67 & 4,67 & 2,89 \\
\hline \multirow{2}{*}{$\begin{array}{l}\text { Aceptación } \\
\text { social }\end{array}$} & $\mathrm{m}$ & 20,55 & 22,57 & 20 , & 17,41 & 21,61 \\
\hline & de & 5,17 & 3,23 & 4,98 & 5,05 & 3,35 \\
\hline \multirow{2}{*}{$\begin{array}{l}\text { Contribu- } \\
\text { ción social }\end{array}$} & $\mathrm{m}$ & 25,61 & 21,15 & 27,16 & 23,02 & 21,94 \\
\hline & de & 3,79 & 2,47 & 3,32 & 3,91 & 2,35 \\
\hline \multirow{2}{*}{$\begin{array}{l}\text { Actualiza- } \\
\text { ción social }\end{array}$} & $\mathrm{m}$ & 24,23 & 23,22 & 23,11 & 20,03 & 24,49 \\
\hline & de & 4,98 & 3,25 & 5,52 & 3,93 & 3,41 \\
\hline \multirow{2}{*}{$\begin{array}{l}\text { Coherencia } \\
\text { social }\end{array}$} & $\mathrm{m}$ & 26,02 & 26,94 & 25,75 & 23,58 & 27,30 \\
\hline & de & 3,95 & 3,06 & 4,58 & 3,95 & 3,33 \\
\hline
\end{tabular}

\section{Correlaciones entre medidas de ideología política e identidad nacional}

Considerando el total de la muestra de los cinco países, las relaciones entre la ideología conservadora dominante $\mathrm{u}$ orientación de la dominación social y las dimensiones de la identidad nacional muestran que solo existe una relación inversa y de baja magnitud entre el grado de identificación con el país y la SDO, $r(905)=-0,07 ; p<0,05$. Sin embargo, un análisis por país de las relaciones entre la SDO y las medidas de identidad nacional sugiere que estas relaciones son más diversas y de mayor magnitud en los casos de las muestras de 
Brasil, Perú y México. Así, en el caso brasileño, la SDO se relaciona inversamente con el grado de identificación con el país, $r(144)=-0,19 ; p<0,05$ $\mathrm{y}$ las dimensiones auto estereotípicas de competencia, $r(143)=-0,31 ; p<0,001$, moralidad, $r(143)$ $=-0,22 ; p<0,01$ y calidez, $r(144)=-0,18 ; p<$ 0,05 . Mientras que en el caso peruano, la SDO se relaciona inversamente con el grado de identificación con el país, $r(220)=-0,25 ; p<0,001$, la autoestima colectiva, $r(220)=-0,19 ; p<0,01 \mathrm{y}$ la dimensión autoestereotípica de competencia, $r(220)=-0,21 ; p<0,01$. Finalmente, en el caso mexicano se aprecia una relación inversa entre la SDO y las dimensiones estereotípicas de competencia, $r(183)=-0,23 ; p<0,01$ y calidez, $r(184)$ $=-0,18 ; p<0,05$.

Por otra parte, las relaciones entre el indicador de autoritarismo de ala derecha (RWA) y las medidas de identidad nacional son variadas para la muestra general de cinco países. Así, la RWA se relaciona positivamente con el grado de identificación con el país, $r(903)=0,22 ; p<0,001$, la autoestima colectiva, $r(974)=0,22 ; p<0,001 \mathrm{y}$ las dimensiones estereotípicas de nacionalismo ideal, $r(977)=0,25 ; p<0,001$, moralidad, $r(956)$ $=0,15 ; p<0,001$ y competencia, $r(960)=0,12$; $p<0,001$. El análisis por países presenta algunas diferencias específicas por países.

Así, en el caso de la muestra chilena, la RWA se relaciona positivamente con todos los indicadores de identidad nacional: grado de identificación con el país, $r(230)=0,30 ; p<0,001$, autoestima colectiva, $r(230)=0,33 ; p<0,001 \mathrm{y}$ las dimensiones auto-estereotípicas de nacionalismo ideal, $r$ (233) $=0,35 ; p<0,001$, moralidad, $r(232)=0,26 ; p<$ 0,001 , competencia, $r(230)=0,23 ; p<0,001 \mathrm{y}$ calidez, $r(229)=0,16 ; p<0,05$. Bajo esta lógica analítica, la muestra colombiana es el segundo grupo donde se aprecian más correlaciones entre la RWA y las dimensiones de la identidad nacional: grado de identificación con el país, $r(197)=0,29$; $p<0,001$, autoestima colectiva, $r(196)=0,31$; $p<0,001$, y las dimensiones auto-estereotípicas de nacionalismo ideal, $r(197)=0,28 ; p<0,001$, moralidad, $r(189)=0,21 ; p<0,001$ y competencia, $r(189)=0,19 ; p<0,05$. Luego, en la muestra peruana se aprecian las siguientes relaciones entre la RWA y las dimensiones de la identidad nacional, autoestima colectiva, $r(223)=0,15 ; p<0,05$ y las dimensiones autoestereotípicas de moralidad, $r(218)=0,20 ; p<0,01$ y nacionalismo ideal, $r(224)$ $=0,17 ; p<0,01$.

Las relaciones entre la RWA y los componentes de la identidad nacional en las muestras de Brasil y México se presentan en menor cantidad. Así, en el caso de Brasil, la RWA se relaciona inversamente con la dimensiones estereotípicas de competencia, $r(140)=-0,27 ; p<0,001$ y calidez, $r(141)=-0,24$; $p<0,01$. Por su parte, en la muestra de México, se aprecian las siguientes relaciones entre la RWA y las dimensiones de la identidad nacional: nacionalismo ideal, $r(183)=0,21 ; p<0,01 \mathrm{y}$ calidez, $r(182)=-0,18 ; p<0,05$

\section{Correlaciones entre medidas de bienestar e identidad nacional}

El análisis de correlación tipo Pearson entre las medidas de satisfacción con la vida y bienestar social para la muestra de los cinco países da como resultado relaciones positivas y significativas entre casi todas las variables analizadas, tal como se muestra en la tabla 10.

Las magnitudes de las relaciones en la muestra general de los cinco países presentan algunas variaciones cuando se analizan las correlaciones de cada país por separado. De manera específica, la muestra peruana es en donde la mayor cantidad de dimensiones identitarias se relacionan positivamente con la satisfacción con la vida, el bienestar social y la mayoría de sus dimensiones. Así, de 42 posibles relaciones, 36 resultan significativas, tal como lo muestra en la tabla 11.

La muestra chilena también presenta diversas asociaciones entre las medidas de bienestar y las dimensiones de la identidad nacional. De manera 
Tabla 10

Correlaciones tipo Pearson entre medidas de bienestar y medidas de identidad nacional en los cinco países

\begin{tabular}{|c|c|c|c|c|c|c|c|}
\hline & $\begin{array}{l}\text { Satisfacción } \\
\text { con la vida }\end{array}$ & $\begin{array}{c}\text { Bienestar } \\
\text { social total }\end{array}$ & $\begin{array}{c}\text { Integración } \\
\text { social }\end{array}$ & $\begin{array}{c}\text { Aceptación } \\
\text { social }\end{array}$ & $\begin{array}{c}\text { Contribución } \\
\text { social }\end{array}$ & $\begin{array}{c}\text { Actualización } \\
\text { social }\end{array}$ & $\begin{array}{c}\text { Coherencia } \\
\text { social }\end{array}$ \\
\hline Grado de identificación & $0,14 * * *$ & $0,37 * * *$ & $0,29 * * *$ & $0,27 * * *$ & $0,18 * * *$ & $0,31 * * *$ & $0,08^{*}$ \\
\hline Autoestima colectiva & $0,26 * * *$ & $0,35 * * *$ & $0,26 * * *$ & $0,26 * * *$ & $0,17 * * *$ & $0,32 * * *$ & $0,12 * * *$ \\
\hline Calidez & $0,11 * * *$ & $0,32 * * *$ & $0,29 * * *$ & $0,13 * * *$ & $0,31 * * *$ & $0,23 * * *$ & $0,11 * * *$ \\
\hline Competencia & $0,15^{* * *}$ & $0,39 * * *$ & $0,29 * * *$ & $0,26 * * *$ & $0,27 * * *$ & $0,30 * * *$ & $0,15 * * *$ \\
\hline Moralidad & $0,10 * *$ & $0,38 * * *$ & $0,25 * * *$ & $0,41 * * *$ & $0,11 * * *$ & $0,29 * * *$ & 0,05 \\
\hline Nacionalismo ideal & $0,11 * * *$ & $0,19 * * *$ & $0,10 * *$ & $0,27 * * *$ & $-0,04$ & $0,18 * * *$ & 0,04 \\
\hline
\end{tabular}

$* * * p<.001, * * p<.01, * p<.05$

Tabla 11

Correlaciones tipo Pearson entre medidas de bienestar y medidas de identidad nacional en la muestra peruana

\begin{tabular}{|c|c|c|c|c|c|c|c|}
\hline & $\begin{array}{l}\text { Satisfacción } \\
\text { con la vida }\end{array}$ & $\begin{array}{c}\text { Bienestar } \\
\text { social total }\end{array}$ & $\begin{array}{l}\text { Integración } \\
\text { social }\end{array}$ & $\begin{array}{l}\text { Aceptación } \\
\text { social }\end{array}$ & $\begin{array}{l}\text { Contribución } \\
\text { social }\end{array}$ & $\begin{array}{l}\text { Actualización } \\
\text { social }\end{array}$ & $\begin{array}{c}\text { Coherencia } \\
\text { social }\end{array}$ \\
\hline $\begin{array}{l}\text { Grado de identifica- } \\
\text { ción }\end{array}$ & 0,09 & $0,37 * * *$ & $0,28 * * *$ & $0,24 * * *$ & $0,14 *$ & $0,36 * * *$ & $0,16^{*}$ \\
\hline Autoestima colectiva & $0,26 * * *$ & $0,36^{* * *}$ & $0,40 * * *$ & $0,26^{* * *}$ & $0,17 * *$ & $0,39 * * *$ & $0,07 * * *$ \\
\hline Calidez & $0,23 * * *$ & $0,33 * * *$ & $0,33 * * *$ & 0,13 & $0,26 * * *$ & $0,26 * * *$ & $0,22 * * *$ \\
\hline Competencia & $0,19 * *$ & $0,42 * * *$ & $0,34 * * *$ & $0,25 * * *$ & $0,31 * * *$ & $0,40 * * *$ & $0,26 * * *$ \\
\hline Moralidad & 0,07 & $0,42 * * *$ & $0,31 * * *$ & $0,43 * * *$ & 0,10 & $0,38 * * *$ & 0,13 \\
\hline Nacionalismo ideal & 0,08 & $0,28 * * *$ & $0,27 * * *$ & $0,18 * *$ & $0,14 *$ & $0,27 * * *$ & $0,28 * * *$ \\
\hline
\end{tabular}

$* * * p<.001, * * p<.01, * p<.05$

específica, de 42 posibles relaciones, 32 resultan significativas, tal como se aprecia en la tabla 12.

La tercera posición, en cuanto a la cantidad de relaciones significativas entre las dimensiones de la identidad y las dimensiones del bienestar, con 27 relaciones significativas de 42 posibles relaciones, la ocupa la muestra colombiana como se aprecia en la tabla 13.

Con 25 relaciones significativas de 42 posibles relaciones entre las dimensiones de identidad y las dimensiones del bienestar se encuentra la muestra brasileña. Los resultados de la muestra brasileña se presentan en la tabla 14 .

Finalmente, con 16 relaciones significativas de 42 posibles relaciones entre las dimensiones de la identidad y las dimensiones del bienestar, se encuentra la muestra mexicana, los resultados de este grupo se presentan en la tabla 15.

\section{Propuesta de un modelo de las relaciones entre la ideología, la identidad nacional y el bienestar}

Los resultados anteriores, sugieren a nivel general influencias de las medidas ideológicas, y especialmente del RWA, en las dimensiones de la identidad nacional. En ese sentido, el rol de la SDO es más limitado para la muestra general de los cinco países. Asimismo, las dimensiones de la identidad se relacionan con las expresiones social y subjetiva del bienestar. Aunque con mayor intensidad en el 
Tabla 12

Correlaciones tipo Pearson entre medidas de bienestar y medidas de identidad nacional en la muestra chilena

\begin{tabular}{lccccccc}
\hline & $\begin{array}{c}\text { Satisfacción } \\
\text { con la vida }\end{array}$ & $\begin{array}{c}\text { Bienestar } \\
\text { social total }\end{array}$ & $\begin{array}{c}\text { Integración } \\
\text { social }\end{array}$ & $\begin{array}{c}\text { Aceptación } \\
\text { social }\end{array}$ & $\begin{array}{c}\text { Contribución } \\
\text { social }\end{array}$ & $\begin{array}{c}\text { Actualización Coherencia } \\
\text { social }\end{array}$ & $\begin{array}{c}\text { Cocial } \\
\text { social }\end{array}$ \\
\hline $\begin{array}{l}\text { Grado de identifi- } \\
\text { cación }\end{array}$ & $0,23 * * *$ & $0,42 * * *$ & $0,36 * * *$ & $31 * * *$ & $0,20 * *$ & $0,31 * * *$ & 0,12 \\
Autoestima colectiva & $0,27 * * *$ & $0,36 * * *$ & $0,31 * * *$ & $0,29 * * *$ & 0,13 & $0,33 * * *$ & 0,12 \\
Calidez & $0,27 * * *$ & $0,35 * * *$ & $0,26 * * *$ & $32 * * *$ & 0,10 & $0,23 * * *$ & $0,14 *$ \\
Competencia & $0,24 * * *$ & $0,43 * * *$ & $0,28 * * *$ & $0,39 * * *$ & $0,15 *$ & $0,36 * * *$ & 0,11 \\
Moralidad & $0,22 * * *$ & $0,42 * * *$ & $0,24 * * *$ & $0,49 * * *$ & 0,04 & $0,38 * * *$ & 0,05 \\
Nacionalismo ideal & $0,26 * * *$ & $0,27 * * *$ & $0,16 *$ & $0,32 * * *$ & 0,08 & $0,27 * * *$ & 0,01 \\
\hline
\end{tabular}

$* * * p<.001, * * p<.01, * p<.05$

Tabla 13

Correlaciones tipo Pearson entre medidas de bienestar y medidas de identidad nacional en la muestra colombiana

\begin{tabular}{|c|c|c|c|c|c|c|c|}
\hline & $\begin{array}{l}\text { Satisfacción } \\
\text { con la vida }\end{array}$ & $\begin{array}{c}\text { Bienestar } \\
\text { social total }\end{array}$ & $\begin{array}{l}\text { Integración } \\
\text { social }\end{array}$ & $\begin{array}{l}\text { Aceptación } \\
\text { social }\end{array}$ & $\begin{array}{c}\text { Contribución } \\
\text { social }\end{array}$ & $\begin{array}{l}\text { Actualización } \\
\text { social }\end{array}$ & $\begin{array}{c}\text { Coherencia } \\
\text { social }\end{array}$ \\
\hline $\begin{array}{l}\text { Grado de identifi- } \\
\text { cación }\end{array}$ & 0,10 & $0,28 * * *$ & $0,22 * *$ & $0,19 * *$ & $0,17 *$ & $0,34 * * *$ & 0,02 \\
\hline Autoestima colectiva & $0,18^{*}$ & $0,30 * * *$ & $0,16^{*}$ & $0,24 * * *$ & $0,18^{*}$ & $0,34 * * *$ & $-0,01$ \\
\hline Calidez & $0,22 * *$ & $0,31 * * *$ & $0,29 * * *$ & $0,23 * * *$ & $0,20 * *$ & $0,15^{*}$ & 0,09 \\
\hline Competencia & $0,22 * *$ & $0,33 * * *$ & $0,26^{* * *}$ & $0,32 * * *$ & $0,20 * *$ & $0,28 * * *$ & 0,02 \\
\hline Moralidad & 0,10 & $0,34 * * *$ & $0,19 * *$ & $0,50 * * *$ & 0,09 & $0,33 * * *$ & $-0,07$ \\
\hline Nacionalismo ideal & 0,08 & $0,27 * * *$ & $0,14 *$ & $0,36 * * *$ & $-0,01$ & $0,33 * * *$ & $-0,07$ \\
\hline
\end{tabular}

$* * * p<.001, * * p<.01, * p<.05$

Tabla 14

Correlaciones tipo Pearson entre medidas de bienestar y medidas de identidad nacional en la muestra brasileña

\begin{tabular}{lccccccc}
\hline & $\begin{array}{c}\text { Satisfacción } \\
\text { con la vida }\end{array}$ & $\begin{array}{c}\text { Bienestar } \\
\text { social total }\end{array}$ & $\begin{array}{c}\text { Integración } \\
\text { social }\end{array}$ & $\begin{array}{c}\text { Aceptación } \\
\text { social }\end{array}$ & $\begin{array}{c}\text { Contribución } \\
\text { social }\end{array}$ & $\begin{array}{c}\text { Actualización Coherencia } \\
\text { social }\end{array}$ \\
\hline social \\
$\begin{array}{l}\text { Grado de identifi- } \\
\text { cación }\end{array}$ & $-0,02$ & $0,45 * * *$ & $0,39 * * *$ & $0,39 * * *$ & $0,25 * *$ & $0,30 * * *$ & 0,02 \\
\hline Autoestima colectiva & 0,06 & $0,41 * * *$ & $0,26 * * *$ & $0,34 * * *$ & $0,20 *$ & $0,34 * * *$ & 0,15 \\
\hline Calidez & 0,04 & 0,10 & 0,08 & 0,10 & 0,07 & $0,21 *$ & 0,02 \\
\hline Competencia & $-0,10$ & $0,38^{* * *}$ & $0,29 * * *$ & $0,37 * * *$ & $0,24 * * *$ & $0,25 * * *$ & $0,19 *$ \\
\hline Moralidad & $-0,09$ & $0,41 * * *$ & $0,31 * * *$ & $0,39 * * *$ & 0,14 & $0,30 * * *$ & 0,10 \\
\hline Nacionalismo ideal & $-0,20 *$ & $0,17 *$ & $0,17^{*}$ & $0,21 *$ & 0,06 & 0,09 & $-0,05$ \\
\hline
\end{tabular}

$* * * p<.001, * * p<.01,{ }^{*} p<.05$ 
Tabla 15

Correlaciones tipo Pearson entre medidas de bienestar y medidas de identidad nacional en la muestra mexicana

\begin{tabular}{lccccccc}
\hline & $\begin{array}{c}\text { Satisfacción } \\
\text { con la vida }\end{array}$ & $\begin{array}{c}\text { Bienestar } \\
\text { social total }\end{array}$ & $\begin{array}{c}\text { Integración } \\
\text { social }\end{array}$ & $\begin{array}{c}\text { Aceptación } \\
\text { social }\end{array}$ & $\begin{array}{c}\text { Contribución } \\
\text { social }\end{array}$ & $\begin{array}{c}\text { Actualización Coherencia } \\
\text { social }\end{array}$ & $\begin{array}{c}\text { Cocial } \\
\text { social }\end{array}$ \\
\hline $\begin{array}{l}\text { Grado de identifi- } \\
\text { cación }\end{array}$ & 0,15 & $0,23^{*}$ & $0,20^{*}$ & $0,25 * *$ & 0,14 & 0,17 & $-0,05$ \\
\hline Autoestima colectiva & $0,33 * * *$ & $0,37 * * *$ & $0,29 * * *$ & $0,21 * *$ & $0,31 * * *$ & $0,24 * * *$ & $0,16^{*}$ \\
\hline Calidez & $-0,02$ & 0,09 & 0,04 & 0,02 & 0,12 & 0,10 & 0,01 \\
\hline Competencia & 0,06 & 0,10 & 0,04 & 0,11 & 0,07 & 0,10 & 0,02 \\
\hline Moralidad & 0,10 & $0,28 * * *$ & $0,17 *$ & $0,39 * * *$ & $-0,05$ & $0,21 * *$ & 0,04 \\
\hline Nacionalismo ideal & 0,04 & $0,16^{*}$ & 0,10 & $0,24 * * *$ & 0,02 & 0,00 & 0,07 \\
\hline$* * * p<.001, * * p<.01, * p<.05$ & & & & & & &
\end{tabular}

primer caso que en el segundo. A continuación, se propone un modelo general de las relaciones entre las dimensiones ideológicas, las dimensiones de la identidad y las expresiones social y subjetiva del bienestar. Por un principio de parsimonia, y atendiendo al hecho de que algunas dimensiones del bienestar social por país no alcanzaron niveles de confiabilidad aceptables, las variables derivadas de estas cinco dimensiones no son incluidas en el análisis de diagramas de ruta.

Inicialmente, se propone un modelo donde las dimensiones ideológicas aparecen como variables exógenas que influyen en primer lugar en los componentes autoestereotípicos de la identidad nacional y luego en la autoestima colectiva y el grado de identificación. Se espera que las influencias del RWA sean positivas y las de la SDO inversas y de menor magnitud. Luego, se espera de manera específica que las dimensiones autoestereotípicas, con excepción de la calidez (Espinosa et al., 2016), influencien en un primer momento a la autoestima colectiva, y en un segundo momento que influencien junto a la autoestima colectiva al grado de identificación nacional. Sobre las relaciones entre las dimensiones identitarias y el bienestar, se esperan influencias directas de los auto-estereotipos de calidez, competencia, moralidad y nacionalismo ideal, así como de la autoestima colectiva y el grado de identificación en el bienestar social. Asimismo, se espera una influencia positiva de la autoestima colectiva y del bienestar social en la dimensión de satisfacción con la vida o bienestar subjetivo. El modelo hipotético propuesto está basado en la revisión teórica inicial y en los resultados correlacionales obtenidos en el presente estudio y se presenta en la figura 1.

Diversos índices fueron utilizados para evaluar la bondad del ajuste de los análisis de senderos propuestos. Entre los que resaltan: el Goodness of Fit Index (GFI) y el Comparative Fit Index (CFI), en los cuales valores sobre 0,90 son considerados niveles aceptables de ajuste del modelo; asimismo, se utilizaron indicadores de error como el Root Mean Square Residual Index (RMR) y el Root Mean Square Error of Approximation (RMSEA) donde valores por iguales o inferiores a 0,08 también indican niveles aceptables de ajuste (Bollen, 1989; Kline, 2005).

El modelo hipotético presenta malos indicadores de ajuste, $\chi^{2}=66,34$, GFI $=0,77$ y CFI $=0,51$, e indicadores de error elevados, $\mathrm{RMR}=0,26 \mathrm{y}$ RMSEA $=0,25$, por lo que su viabilidad estadística no puede ser aceptada. En ese sentido, un modelo alternativo es propuesto. El nuevo modelo descarta la SDO por presentar pocas influencias significativas en las variables identitarias. Asimismo, retira 


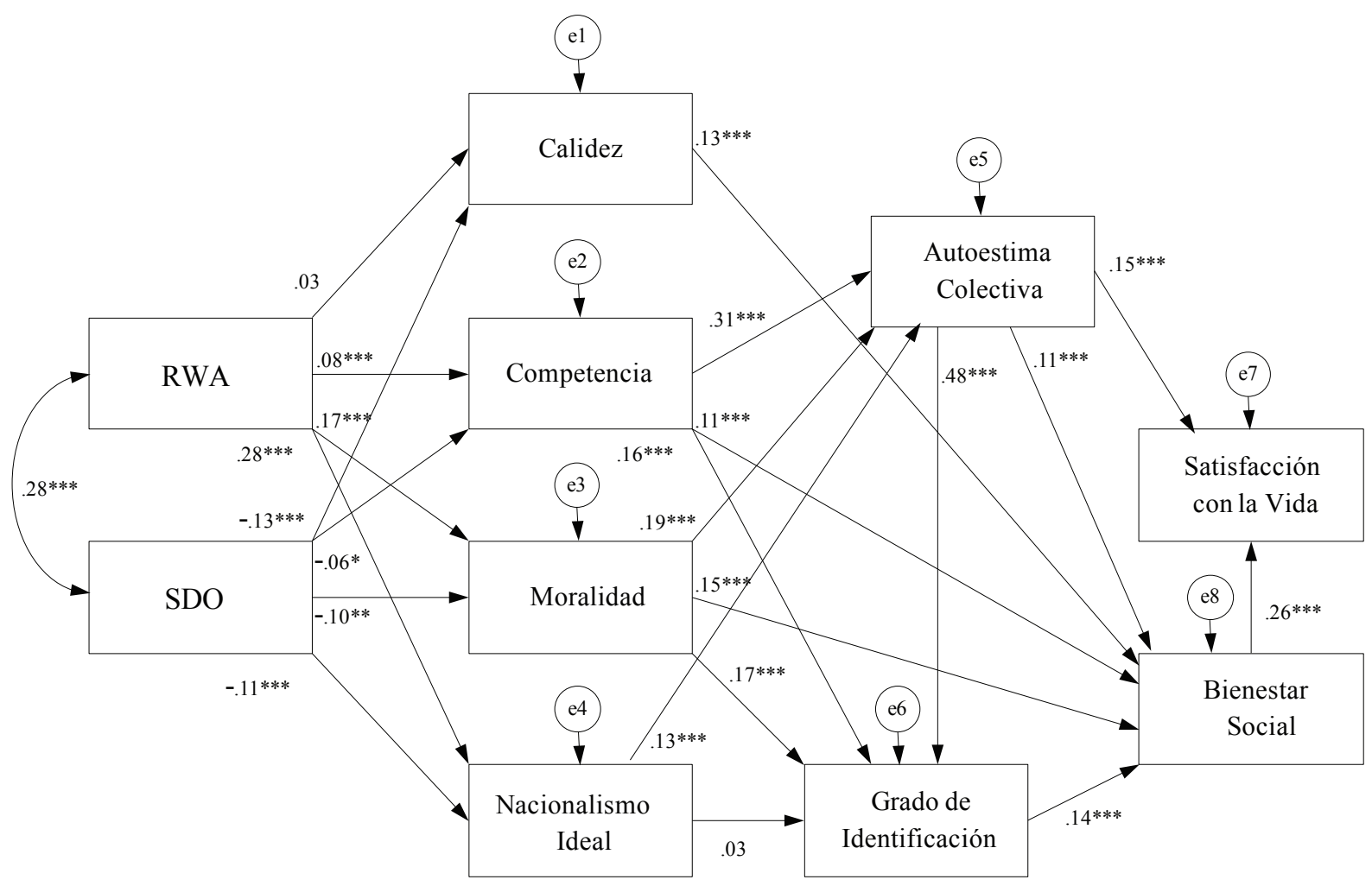

Figura 1. Diagrama de las relaciones entre las medidas ideológicas, las dimensiones de la identidad nacional y las dimensiones del bienestar (Modelo hipotético)

$* * * p<.001, * * p<.01, * p<.05$

del análisis la dimensión de calidez por razones similares. El modelo alternativo presenta entonces el RWA como variable exógena, que influye directamente en las dimensiones auto-estereotípicas, la autoestima colectiva y el grado de identificación. Las dimensiones autoestereotípicas por su parte influyen en la autoestima colectiva. La autoestima colectiva y las dimensiones autoestereotípicas también influirán en el grado de identificación con el país. Asimismo, las medidas identitarias ingresadas en el análisis tienen una influencia directa en el bienestar social. Finalmente, la autoestima colectivo y el bienestar social tienen una influencia positiva en el bienestar subjetivo, evaluado con el indicador de satisfacción con la vida. El modelo alternativo se presenta en la figura 2.
Los resultados del modelo alternativo, aunque mejoran estadísticamente, también presentan indicadores de ajuste inadecuados, $\chi^{2}=56,68, \mathrm{GFI}=$ 0,84 y CFI $=0,63$, e indicadores de error elevados, $\mathrm{RMR}=0,24$ y RMSEA $=0,23$, por lo que la viabilidad del modelo alternativo para las muestras de los cinco países resulta también en términos estadísticos, inapropiado.

\section{Discusión}

Atendiendo al primer objetivo del estudio que buscaba describir y analizar las relaciones entre las ideologías conservadoras autoritaria y dominante con las dimensiones de la identidad nacional, se encontró que se avala la afirmación general acerca 


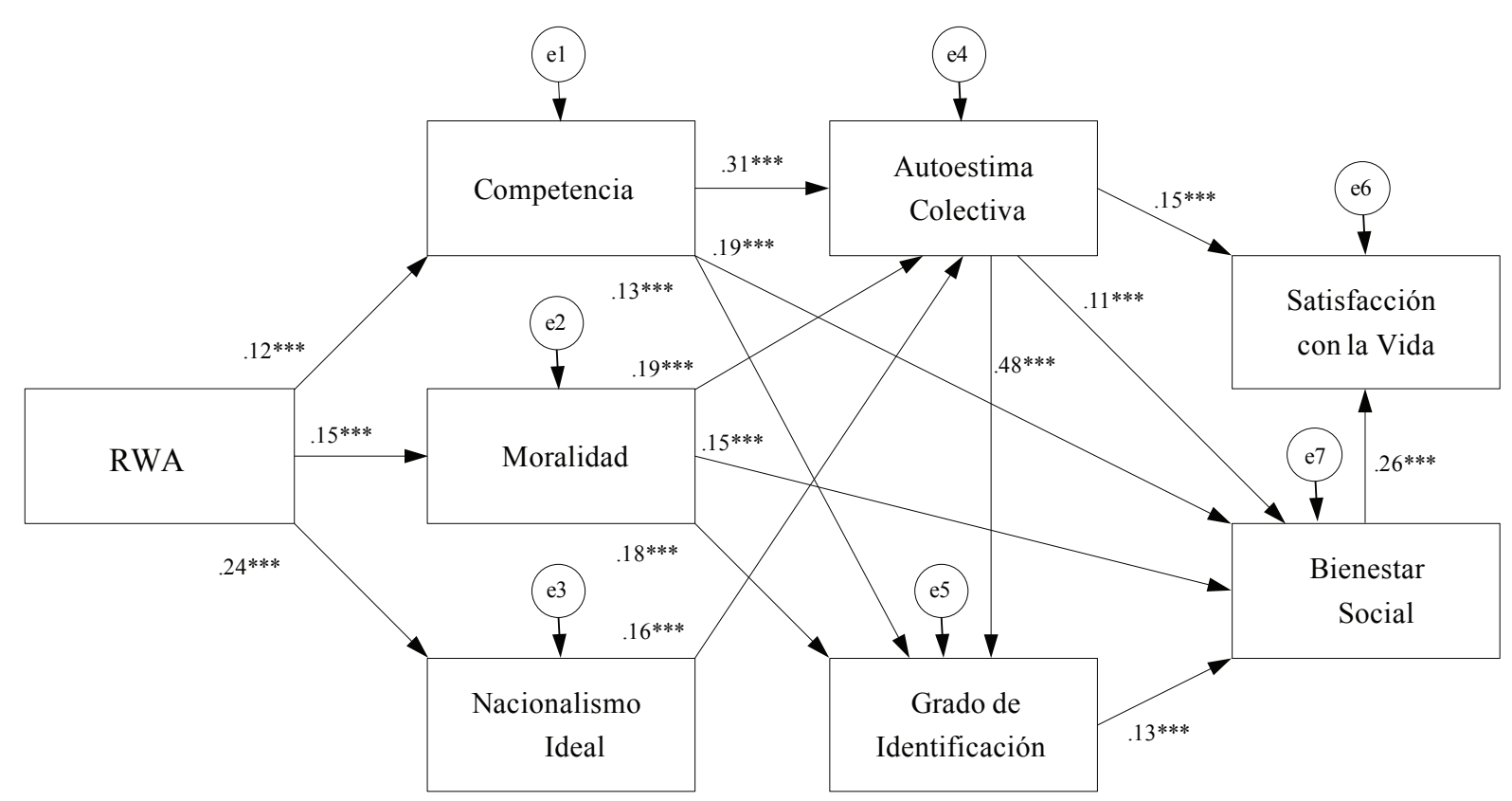

Figura 2. Diagrama de las relaciones entre las medidas ideológicas, las dimensiones de la identidad nacional y las dimensiones del bienestar (Modelo alternativo)

${ }^{* * *}{ }_{p}<.001,{ }^{* *} p<.01,{ }^{*} p<.05$

de que las representaciones identitarias que comprenden la categoría nacional están estrechamente relacionadas con posiciones ideológicas de naturaleza conservadora. Es sobre este punto que se aprecia el aporte de la psicología política al estudio de las identificaciones nacionales; pues, por una parte, la ideología conservadora autoritaria, como era de esperarse, se relacionó positivamente con algunos componentes de la identidad nacional en cuatro de los cinco países. Estas relaciones son especialmente constantes en la muestra chilena y colombiana, donde se podría apreciar con mayor claridad la idea de que la ideología conservadora promueve la identificación con una categoría nacional por medio de elementos que estimulan la cohesión grupal (Smith, 2004). Desde esta perspectiva, es razonable encontrar que el RWA se haya relacionado positivamente con un conjunto de contenidos autoestereotípicos positivos en las muestras chilena, colombiana y peruana, ya que, como refieren Van Vugt y Hart (2004), las expectativas positivas sobre un endogrupo refuerzan la cohesión en su interior. En esta misma línea, Smith et al. (2005) encuentran que la autoestereotipia positiva refuerza la identificación nacional en muestras de varios países europeos. Ahora bien, los aspectos autoestereotípicos que parecen ensalzarse a partir de una posición ideológica autoritaria están ligados a las dimensiones con un mayor contenido cívico y moral: nacionalismo ideal y moralidad. Esto es interesante en la medida en que en América Latina se reportan sistemáticamente problemas de relaciones interpersonales e intergrupales ancladas en la transgresión y la desconfianza (Beramendi, 2014), lo que parece exacerbar la necesidad de conceptualizar - al menos desde una representación ideológico-identitaria - a los compatriotas como personas cívicas, patriotas y dignas de confianza. Este hecho parece relacionarse con la idea de que los mitos nacionalistas en ocasiones distorsionan la realidad (Gellner, 2008) y explica la aparente contradicción entre la definición propuesta por 
Espinosa et al., (2016) sobre la dimensión autoestereotípica de nacionalismo ideal en términos de una representación de conciudadanos comprometidos con el país, críticos con lo negativo que en este acontece y desarrollados, así como con la expectativa de que esta dimensión se relacione de manera inversa con la ideología nacionalista autoritaria, cuando en el presente estudio acontece lo opuesto.

De manera particular, el caso de la muestra brasileña es interesante, porque las relaciones entre el RWA y algunas dimensiones identitarias son inversas. Es difícil proveer una explicación concreta al comportamiento estadístico de la muestra brasileña. Sin embargo, es importante tomar en consideración que es en este grupo donde la relación entre el RWA y la SDO ha sido más intensa. Lo anterior podría haber originado un problema de multicolinealidad y el comportamiento del RWA para este caso concreto se asemeja al comportamiento de la SDO.

Es entonces que se discute un segundo aporte de la psicología política al estudio de la identificación nacional, y es el de las relaciones entre la ideología conservadora dominante y los procesos de identificación nacional en algunas muestras latinoamericanas. Sobre este punto, estudios previos en Argentina (Monsegur et al., 2014) y Perú (Espinosa, 2010) dan cuenta de relaciones inversas entre la SDO y algunos componentes de la identidad nacional en estos países. Algo similar ocurre en el presente estudio con las muestras brasileña, mexicana y peruana. Lo anterior parece corroborar lo propuesto por Levin y Sidanius (1999), quienes afirman que las personas que presentan una orientación hacia la dominancia social suelen expresar valores y afectos negativos hacia los grupos de bajo estatus, incluso cuando se trata del endogrupo. Como se ha referido, lo anterior podría estar vinculado con el fenómeno de altercentrismo que ha caracterizado a las naciones de América Latina (Montero, 1992).
Las muestras chilena y colombiana no dan cuenta de relaciones de ninguna índole entre la SDO y los componentes de la identidad nacional. Lo anterior sugiere que el cuestionamiento sobre el estatus relativo de la categoría nacional no es un problema psicopolítico relevante en las muestras de estos países, por lo que la representación positiva del endogrupo nacional seguirá una ruta ideológica autoritaria que no se verá afectada por la ideología de dominación social. La relación entre la identificación y el autoritarismo en la muestra chilena podría estar relacionada con aquello que Hosiasson (2011) identifica como un espíritu nacionalista reaccionario que gravita en las reflexiones de los chilenos con respecto a su historia.

En cuanto al segundo objetivo del presente estudio, que buscaba describir y analizar las relaciones entre las expresiones del bienestar subjetivo y social con los componentes de la identidad nacional, se puede afirmar que existe evidencia de que la categoría nacional para los participantes de Chile, Colombia y Perú garantizan un proceso de identificación que se vincula claramente al bienestar. Lo anterior, no resulta tan claro para los casos de las muestras brasileña y mexicana, aunque tampoco se puede descartar de plano para estas, ya que un estudio en México da cuenta de relaciones significativas entre el grado de identificación con el endogrupo nacional mexicano y las dimensiones del bienestar social en una muestra de estudiantes universitarios de ese país (Laca-Arocena et al., 2010).

Este último resultado, lejos de desacreditar las propuestas conceptuales que sugieren que la identidad y el bienestar se encuentran ligados, invitan a pensar si en estos casos las categorías nacionales son fuentes de bienestar o si son otras adscripciones identitarias las que satisfacen esta necesidad. También se abre un espacio para discutir los elementos sobre los que se pueden construir narrativas identitarias en Brasil y México que permitan relacionar más intensamente la 
identificación con el endogrupo nacional a experiencias de bienestar.

En mayor o menor medida, los componentes de la identidad nacional se relacionan con las expresiones del bienestar estudiadas y, de manera consistente con estudios previos en Perú, se encuentra que las relaciones entre los componentes de la identidad nacional, el bienestar social y sus dimensiones son de mayor magnitud que la relaciones entre los componentes de la identidad nacional y el bienestar subjetivo (Espinosa \& Tapia, 2011; León, 2011). Espinosa y Tapia (2011) sugieren que este efecto estadístico corresponde al hecho de que tanto la identidad nacional como el bienestar social suponen una apreciación subjetiva de un escenario psicológico arraigado en lo colectivo, mientras que el bienestar subjetivo corresponde a una representación vinculada con un plano más individualizado. Por tal motivo, la identificación nacional como un subtipo de identificación colectiva y el bienestar social se asociarán de manera más intensa, dado que existe una correspondencia mayor entre ellas.

De manera específica, los aspectos del bienestar más asociados con los componentes de la identidad nacional son heterogéneos por país. Sin embargo, las dimensiones que parecen ser más importantes son la integración social, la aceptación social y la actualización social. Es decir, aquellas que se relacionan con expectativas concretas de obtención de algún beneficio del contexto social en que las personas se desenvuelven, antes que la inversión en este. Así, la integración social es una medida de inclusión social y da cuenta del sentido de pertenecer a un grupo social en un contexto determinado, resultando casi una medida de identidad en sí misma (Espinosa, 2010).

La aceptación social, por su parte, puede ser entendida como una medida de confianza interpersonal, es decir, cuánto es posible confiar en los miembros del grupo social al que se pertenece y resulta interesante que en todas las muestras por país, la relación entre esta dimensión y el auto- estereotipo de moralidad presente de las mayores magnitudes estadísticas observadas a nivel general en el estudio. Finalmente, la actualización social da cuenta de las posibilidades de crecimiento personal que, se percibe, el contexto social podría proveer. Por otra parte, las relaciones de las dimensiones del contribución y coherencia social con las dimensiones identitarias son menos frecuentes e intensas en las muestras por país, lo que refuerza la idea de que las personas constituyen su bienestar sobre la base de la obtención de beneficios concretos anclados en la inclusión, la confianza y las oportunidades de crecimiento personal y social.

El hecho de que los resultados confirmen una relación entre algunas dimensiones de la identificación nacional y las expresiones del bienestar evaluadas invita a reflexionar sobre la necesidad de promover estrategias de refuerzo de dichas identificaciones en los países evaluados, pues estas podrían tener un impacto en el bienestar de las personas, especialmente en la sensación de integración social, en una mayor confianza interpersonal y en las expectativas de un mayor desarrollo personal y social.

En cuanto al tercer objetivo del presente estudio, se proponía encontrar un modelo general de las relaciones entre la ideología, la identidad y el bienestar. La propuesta de un modelo teórico hipotético y la evaluación de uno alternativo más parsimonioso no resultó adecuada en términos estadísticos. Algunas posibles explicaciones a estos resultados se basan en el hecho de que las representaciones identitarias responden a un fenómeno contextualizado (Beramendi, 2014), y, si bien hay algunos elementos en común en los procesos sociales y políticos que viven los países de América Latina, también hay procesos que los diferencian y que explicarían la relativa heterogeneidad de los resultados obtenidos en las muestras de los cinco países. Esta heterogeneidad, sin duda, va a estar anclada en la diversidad étnica y social de estos países y en los momentos políticos que atraviesan en la actualidad. La heterogeneidad de los resul- 
tados también va a estar relacionada con el hecho de que las muestras no son representativas de la población general de los países en que fueron tomadas y, al tratarse de muestreos intencionales, estas presentan unas diferencias sociales importantes.

Lo anterior permite afirmar que, aún cuando existen relaciones claras entre la identidad nacional, las ideologías conservadoras y las expresiones del bienestar, un análisis detallado de estas relaciones no permite desarrollar un modelo general con buenos niveles de ajuste estadístico para las muestras de los cinco países de América Latina que han sido estudiadas. Esto parece ocurrir por las diferencias específicas por país que se encuentran en algunas relaciones entre las variables estudiadas.

Trascendiendo los objetivos de la investigación, la presente investigación reporta algunos hallazgos interesantes y consistentes con algunos estudios previos. Así, se observa que la dimensión autoestereotípica más consensual en las cinco muestras fue la calidez (Espinosa et al., 2016). Sin embargo, no se consigue apreciar una influencia directa de esta, ni en el grado de identificación con el país ni en la autoestima colectiva ni con ninguna de las dimensiones del bienestar. Esto llama la atención porque el atributo estereotípico positivo más predominante en los países de América Latina no consigue satisfacer con claridad ningún motivo identitario ni ningún criterio psicosocial de bienestar. En ese sentido, la propuesta de intervención que se desprende del presente estudio debe relacionar el desarrollo de los discursos identitarios en las dimensiones de moralidad, el nacionalismo ideal y la competencia. Sin embargo, como se ha descrito previamente, en términos de lo propuesto por Gellner (2008), esto podría suponer un riesgo de distorsión basado en una narrativa de la exaltación nacionalista que se base en discursos moralistas y cívicos maniqueos, antes que en una propuesta coherente de desarrollo de la identificación con los endogrupos nacionales, realmente inclusiva y respetuosa de las diferencias que caracterizan a las naciones de América Latina.

\section{Referencias}

Abrams, D., \& Hogg, A. (1990). An introduction to the social identity approach. En: D. Abrams \& M. A. Hogg (Eds.). Social Identity Theory: Constructive and Critical Advances (pp. 1-9). New York: Harvester Wheatsheaf.

Altemeyer, B. (2004). The other "authoritarian personality”. En J. T. Jost \& J. Sidanius (Eds.), Political psychology (pp. 85-107). New York: Psychology Press.

Anderson, B. (1993). Comunidades Imaginadas: Reflexiones sobre el origen y la difusión del nacionalismo. México, D.F.: Fondo de Cultura Económica

Bar-Tal, D. (1996). Las creencias grupales como expresión de identidad social. En J. F. Morales, D. Páez, J. C. Deschamps \& S. Worchel (Eds.). Identidad social. Aproximaciones psicosociales a los grupos y a las relaciones entre grupos (pp. 255-286). Valencia: Promolibro.

Béjar, R., \& Cappello, H. M. (1986). La identidad y carácter nacionales en México - La frontera de Tamaulipas. Revista de Psicología Social, 1, 153-166.

Beramendi, M. (2014). Percepción del sistema normativo, transgresión y sus correlatos psicosociales en Argentina. (Tesis doctoral no publicada, Facultad de Psicología, Universidad de Buenos Aires, Ciudad Autónoma de Buenos Aires, Argentina).

Beramendi, M., \& Zubieta, E. (2013). Identidad nacional y relaciones interpersonales en una cultura donde la norma es la transgresión. Psicología Política, 26, 165-177.

Blanco, A., \& Díaz, D. (2005). El Bienestar Social: su concepto y medición. Psicothema, 17(4), 582-589.

Blank, T. (2003). Determinants of National Identity in East and West Germany: An Empirical Comparison of Theories on the Significance of Authoritarianism, Anomie and General Self-Esteem. Political Psychology, 24 (2), 259-288. 
Bollen, K. A. (1989). Structural equations with latent variables. New York, N.Y.: John Wiley \& Sons.

Cárdenas, M., Meza, P., Lagues, K., \& Yáñez, S. (2010). Adaptación y validación de la escala de orientación a la dominancia social (SDO) en una muestra chilena. Universitas Psychologica, 9(1), 161-168.

Cárdenas, M., \& Parra, L. (2010). Adaptación y validación de la Versión Abreviada de la Escala de Autoritarismo de Derechas (RWA) en una muestra chilena. Revista de Psicología, 19(1), 61-79.

Carteri, K. K. (2008). A identidade nacional brasilei$\mathrm{ra}$. Recuperado de: http://biblioteconomiaepatrimonio.blogspot.com/2008/07/identidade-nacional-brasileira-karin.html

D’Adamo, O., \& García-Beaudoux, V. (1995). El argentino feo. Ciudad Autónoma de Buenos Aires: Losada.

Dekker, H., Malová, D., \& Hoogendoorn, S. (2003). Nationalism and Its Explanations. Political Psychology, 24, 345-376.

Diener, E., Emmons, R., Larsen, R., \& Griffin, S. (1985). The Satisfaction with Life Scale. The Journal of Personality Assessment, 49, 71-75.

Espinosa, A. (2003). Identidad social e identidad nacional en una muestra de triciclistas en Juliaca. (Tesis de Licenciatura no publicada, Pontificia Universidad Católica del Perú, Lima, Perú).

Espinosa, A. (2010). Estudios sobre Identidad Nacional en el Perú y sus correlatos psicológicos, sociales y culturales. (Tesis doctoral no publicada, Universidad del País Vasco, San Sebastián).

Espinosa, A., Acosta, Y., Valencia, J., Vera, A., Soares-da Silva, A., Romero, J. C., \& Beramendi, M. (2016). Calidez, Competencia, Moralidad y Nacionalismo ideal como dimensiones del autoconcepto nacional en seis países de Latinoamérica. Avances en Psicología Latinoamericana, 34(2), 395-412.

Espinosa, A., \& Beramendi, M., (2012). Diseño y evaluación de la Escala de Autoestereotipos $\mathrm{Na}$ cionales Argentinos. Manuscrito no publicado.
Espinosa, A., Beramendi, M., \& Zubieta, E. (2012). ¿Naciones saludables? Identidad nacional como fuente de bienestar subjetivo y social: Una sintesis meta-analítica de estudios en Argentina, México y Perú. Trabajo presentado en el IV Congreso Latino-Americano de la ULAPSI, Montevideo.

Espinosa, A., \& Cueto, R. M. (2014). Estereotipos raciales, racismo y discriminación en América Latina. En E. Zubieta, J. Valencia \& G. Delfino (Coords.). Psicología Social y Política: Procesos teóricos y estudios aplicados (pp. 352-361). Buenos Aires: EUDEBA.

Espinosa, A., \& Tapia, G. (2011). Identidad nacional como fuente de bienestar subjetivo y social. Boletín de Psicología, 102, 71-87.

Feshbach, S. (1987). Individual Aggression, National Attachment, and the Search for Peace: Psychological Perspectives. Aggressive Behavior, 13, 315-325.

Gellner, E. (2008). Nations and nationalism. New York: Cornell University Press.

Haslam, S. A., Jetten, J., Postmes, T., \& Haslam, C. (2009). Social identity, health and well-being: An emerging agenda for applied psychology. Applied Psychology: An International Review, 58, 1-23.

Herranz, K., \& Basabe, N. (1999). Identidad nacional, ideología política y memoria colectiva. Psicología Política, 18, 31-47.

Hogg, M. A., \& Abrams, D. (1988). Social identifications: A social psychology of intergroup relations and group processes. London: Routledge.

Hosiasson, L. J. (2011). Nação e Imaginação na Guerra do Pacífico. São Paulo: EDUSP.

Hurwitz, J., \& Peffley, M. (1999). International Attitudes. En J. P. Robinson, P. R. Shaver \& L.S. Wrightsman (Eds.). Measures of Political Attitudes (pp. 533-590). Academic Press: Boston.

Kaldor, M. (2004). Nationalism and Globalisation. Nations and Nationalism, 10(1/2), 161-177.

Keillor, B., Tomas, G., \& Hult, M. (1999). A five-country study of national identity. Implications for 
international marketing research and practice. International Marketing Review, 16, 65-82.

Keyes, C. (1998). Social Well-being. Social Psychology Quarterly, 61(2), 121-140.

Kline, R. B. (2005). Principles and practice of structural equation modeling (2. ${ }^{\mathrm{a}}$ ed.). New York, N.Y.: Guilford Press.

Laca-Arocena, F., Mejía, J. C., \& Yáñez, C. (2010). Identidad mexicana e interés político: Predictores de bienestar social y anomia. Acta Universitaria, 20(2), 40-49.

León, E. (2012). Percepción del contexto nacional y su relación con el clima emocional, el bienestar y la identidad nacional en una muestra de estudiantes de Cajamarca, Lima y Pucallpa. (Tesis de Licenciatura no publicada, Pontificia Universidad Católica del Perú, Lima, Perú).

Levin, S., \& Sidanius, J. (1999). Social dominance and social identity in the United States and Israel: Ingroup favoritism or outgroup derogation? Political Psychology, 20, 99-126.

Luhtanen, R., \& Crocker, J. (1992). A Collective Self-esteem Scale: Self Evaluation of one's Identity. Personality and Social Psychology Bulletin, 18, 302-318.

Lyons, A. C., Cude, B., Lawrence, F. C., \& Gutter, M. (2005). Conducting Research Online: Challenges Facing Researchers in Family and Consumer Sciences. Family and Consumer Sciences Research Journal, 33, 341-356.

Martínez, P. (2006). Perspectiva futura del Perú y nivel de satisfacción con él. Liberabit, 12, 113121.

Mezulis, A. H., Abramson, L. Y., Hyde, J. S., \& Hankin, B. L. (2004). Is there a universal positivity bias in attributions? A meta-analytic review of individual, developmental and cultural differences in the self-serving attributional bias. Psychological Bulletin, 130, 711-747.

Miles, R. (2000). Nacionalismo. En E. Cashmore. (Ed.). Dicionário de relações étnicas e raciais (pp. 383-386). São Paulo: Selo Negro.
Monsegur, S, Espinosa, A., \& Beramendi, M. (2014). Identidad Nacional y su relación con la Dominancia Social y la Tolerancia a la Transgresión en residentes de Buenos Aires (Argentina). Interdisciplinaria, 31, 5-21.

Montero, M. (1992). Atracción y repulsión: Identidad nacional en hijos de inmigrantes. Boletín de Psicología, 37, 21-42.

Montero, M. (1996). Identidad social negativa y crisis socioeconómica: un estudio psicosocial. Revista Interamericana de Psicología, 30, 43-85.

Morales, J. F., \& Páez, D. (1996). Estereotipos, discriminación y relaciones intergrupos en España y en Latinoamérica. En R. Y. Bourhis, J-P. Leyens, J. F. Morales \& D. Páez (Eds.). Estereotipos, discriminación y relaciones entre grupos (pp. 1-22). Madrid: McGraw-Hill.

Morrison, M., Tay, L., \& Diener, E. (2010). Subjective Well-Being and National Satisfaction: Findings from a Worldwide Survey: Psychological Science, 22(2), 166-171.

Nigbur, D., \& Cinnirella, M. (2007). National identification, type and specificity of comparison and their effects on descriptions of national character. European Journal of Social Psychology, 37, 672-691.

Pérez de León, P. (2007). Identidades, actitudes y estereotipos nacionales y supranacionales en una muestra uruguaya. Ciencias Psicológicas, 1(1), 81-102.

Pérez, T. (1999). Nación, identidad nacional y otros mitos nacionalistas. Oviedo: Ediciones Nobel.

Pires, A. M. L. T. (2010). El prejuicio racial en Brasil: medidas comparativas. Psicologia \& Sociedade, 22, 32-42.

Pratto, J., Sidanius, J., Stallworth, L., \& Malle, B. (1994). Social dominance orientation: A personality variable predicting social and political attitudes. Journal of Personality and Social Psychology, 67, 741-763.

Richey, S. (2011). Civic Engagement and Patriotism. Social Science Quarterly, 92(4), 1044-1056. 
Roccatto, M., \& Sclauzero, M. (2002). Autoritarismo, nacionalismo y patriotismo. Un estudio con militantes italianos. Psicología Política, 25, 21-36.

Rodríguez-Carvajal, R., Díaz, D., Moreno-Jiménez, B., Blanco, A., \& van Dierendock, D. (2010). Vitalidad y recursos internos como componentes del constructo de bienestar psicológico. Psicothema, 22(1), 63-70.

Roselli, N. (2000). Representación social de "los argentinos", "los españoles", "los latinoamericanos" y "los europeos" en estudiantes universitarios argentinos. Revista Latinoamericana de Psicología, 32(1), 127-160.

Salazar, J. M. (1996). Identidad social e identidad nacional. En J. F. Morales, D. Páez, J. C. Deschamps \& S. Worchel (Eds.). Identidad social. Aproximaciones psicosociales a los grupos y a las relaciones entre grupos (pp. 495-515). Valencia: Promolibro.

Salazar, J. M., \& Salazar, M. A. (1998). Estudios recientes acerca de identidades nacionales en América Latina. Psicología Política, 16, 75-93.

Simon, B. (2004). Identity in a modern society: A social psychological perspective. Oxford: Blackwell.
Smith, A. D. (2004). Nacionalismo. Madrid: Alianza Editorial.

Smith, P. B., Giannini, M., Helkama, K., Maczynski, J., \& Stumpf, S. (2005). Positive Auto-stereotyping and Self-construal as Predictors of National Identification. International Review of Social Psychology, 18, 65-90.

Tajfel, H. (1984). Grupos humanos y categorías sociales: Estudios de psicología social. Barcelona: Herder.

Van Vugt, M., \& Hart, C. M. (2004). Social identity as social glue: The origins of group loyalty. Journal of Personality and Social Psychology, 86, 585-598.

Vignoles, V. L., Regalia, C., Manzi, C., Golledge, J., \& Scabini, E. (2006). Beyond self-esteem: Influence of multiple motives on identity construction. Journal of Personality and Social Psychology, 90, 308-333.

Villas-Bôas, L. P. S. (2010). Brasil. Ideia de diversidade e representações sociais. São Paulo: Anna Blume.

Vives, J. (1994). Identidad Mexicana: Un Proceso con Cinco Siglos de Duración. Psicología Iberoamericana, 2(3), 14-20. 